\title{
Utilization of a Directly Supervised Telehealth-Based Exercise Training Program in Patients With Nonalcoholic Steatohepatitis: Feasibility Study
}

Victoria Motz, MD; Alison Faust, MD; Jessica Dahmus, MD; Benjamin Stern, DO; Christopher Soriano, MD; Jonathan G Stine, MD, MSc

Penn State Milton S Hershey Medical Center, Hershey, PA, United States

\section{Corresponding Author:}

Jonathan G Stine, MD, MSc

Penn State Milton S Hershey Medical Center

500 University $\mathrm{Dr}$

Hershey, PA, 17033

United States

Phone: 17175311017

Email: jstine@pennstatehealth.psu.edu

\begin{abstract}
Background: Most patients with nonalcoholic fatty liver disease (NAFLD) are physically inactive despite the well-known benefits of physical activity. Telehealth offers promise as a novel way to deliver an exercise training program and increase physical activity. However, the feasibility, safety, and efficacy of telehealth-based exercise programs is unknown in patients with NAFLD.

Objective: The aim of this study was to determine the feasibility of a directly supervised exercise training program delivered exclusively with telehealth to patients with nonalcoholic steatohepatitis (NASH), the progressive form of NAFLD.

Methods: In response to COVID-19 research restrictions, we adapted an existing clinical trial and delivered 20 weeks of moderate-intensity aerobic training 5 days a week under real-time direct supervision using an audio-visual telehealth platform. Aerobic training was completed by walking outdoors or using a home treadmill. Fitness activity trackers with heart rate monitors ensured exercise was completed at the prescribed intensity with real-time feedback from an exercise physiologist.

Results: Three female patients with biopsy-proven NASH were enrolled with a mean age of 52 (SD 14) years. The mean body mass index was 31.9 (SD 5.1) kg/m2. All patients had metabolic syndrome. All patients completed over $80 \%$ of exercise sessions (mean 84\% [SD 3\%]) and no adverse events occurred. Body weight (mean $-5.1 \%$ [SD 3.7\%]), body fat (mean $-4.4 \%$ [SD 2.3\%]), and waist circumference (mean $-1.3 \mathrm{in}$. [SD $1.6 \mathrm{in}$.$] ) all improved with exercise. The mean relative reduction in magnetic resonance$ imaging-proton density fat fraction (MRI-PDFF) was 35.1\% (SD 8.8\%). Mean reductions in hemoglobin A1c and Homeostatic Model Assessment for Insulin Resistance were also observed ( $-0.5 \%$ [SD $0.2 \%$ ] and -4.0 [SD 1.2], respectively). The mean peak oxygen consumption (VO2peak) improved by 9.9 (SD 6.6) $\mathrm{mL} / \mathrm{kg} / \mathrm{min}$.
\end{abstract}

Conclusions: This proof-of-concept study found that supervised exercise training delivered via telehealth is feasible and safe in patients with NASH. Telehealth-based exercise training also appears to be highly efficacious in patients with NASH, but this will need to be confirmed by future large-scale trials.

Trial Registration: ClinicalTrials.gov NCT03518294; https://clinicaltrials.gov/ct2/show/NCT03518294

(JMIR Form Res 2021;5(8):e30239) doi: 10.2196/30239

\section{KEYWORDS}

physical activity; fatty liver; telemedicine; liver; nonalcoholic fatty liver disease; liver disease; fatty liver disease; aerobic training; telehealth; fitness; feasibility; steatohepatitis

\section{Introduction}

To date, there is no effective drug therapy nor cure for nonalcoholic fatty liver disease (NAFLD) or its progressive form, nonalcoholic steatohepatitis (NASH). Lifestyle modification, which includes both dietary change and increasing physical activity, remains the most effective treatment for NAFLD and is recommended for all patients. Despite physical activity's well-known benefits, over $80 \%$ of patients with 
NAFLD are physically inactive [1,2]. Consequently, disease progression is common. There is a clear unmet need to increase physical activity in order to improve patient outcomes, especially in light of the rapidity of weight gain from increased sedentary behavior attributable to the novel COVID-19 pandemic [3]. Out of necessity, telehealth has emerged at the forefront of health care delivery during the pandemic. Telehealth offers additional promise for patients with NAFLD to (1) remove self-identified barriers preventing physical activity [1] and (2) deliver an exercise training program. However, telehealth-delivered exercise training programs remain unexplored in patients with NAFLD and their feasibility, safety, and efficacy are unknown.

\section{Methods}

Because institutional restrictions prevented in-person clinical trial activity at the height of the pandemic, we adapted an existing clinical trial that was actively recruiting patients (prior to COVID-19 restrictions, 25 patients were recruited under the original clinical trial protocol) [4] and delivered 20 weeks of moderate-intensity aerobic training 5 days a week under real-time direct supervision by an exercise physiologist using an Institutional Review Board-approved audio-visual (A-V) telehealth platform with 2-way communication. Aerobic training was completed by walking outdoors or using a home treadmill. Fitness activity trackers with heart rate monitors ensured exercise was completed at the prescribed intensity with real-time feedback from an exercise physiologist. Each exercise session lasted 30 minutes, and was preceded by a warm-up and ended with a cool-down in accordance with the original study protocol.
Feasibility was defined as completing $80 \%$ or more of exercise sessions [5]. Secondary clinical outcomes were captured according to the existing study protocol [4]. Patients also received dietary counseling according to the original study protocol which allowed for telehealth as a way to provide the nutritional feedback.

\section{Results}

Three patients with biopsy-proven NASH were recruited and enrolled during the period of COVID-19 research restrictions. All patients were female with a mean age of 52 (SD 14) years. The mean body mass index was 31.9 (SD 5.1) kg/m². All patients had metabolic syndrome. Liver histology was as follows: NAFLD activity score of $4(n=2)$ and $5(n=1)$; and fibrosis stage of $1(n=2)$ and $0(n=1)$. All patients completed $80 \%$ or more of exercise sessions (mean $84 \%$ [SD 3\%]) and no adverse events occurred. Body weight (mean $-5.1 \%$ [SD 3.7\%]), body fat (mean $-4.4 \%$ [SD 2.3\%]), and waist circumference (mean -1.3 [SD 1.6] in.) all improved with exercise (Figure 1). The mean relative reduction in liver fat measured by magnetic resonance imaging-proton density fat fraction (MRI-PDFF) was $35.1 \%$ (SD 8.8\%). Mean reductions in hemoglobin A1c, aspartate aminotransferase, alanine aminotransferase, Homeostatic Model Assessment for Insulin Resistance were $-0.5 \%$ (SD 0.2\%), -8.5 (SD 3.2) IU/L, -12.5 (SD 6.7) IU/L, and -4.0 (SD 1.2), respectively. The mean peak oxygen consumption $\left(\mathrm{VO}_{2}\right.$ peak) improved by 9.9 (SD 6.6) $\mathrm{mL} / \mathrm{kg} / \mathrm{min}$. Owing to the small sample size, no change in secondary clinical outcomes achieved statistical significance $(P>.05)$. 
Figure 1. Observed clinical benefits of a supervised 20-week telehealth-delivered exercise training program. (A) All patients reduced MRI-PDFF-measured liver fat with exercise training. (B) Exercise training reduced body weight in all patients. (C) Cardiorespiratory fitness improved in all patients (mean VO2 peak +9.9 [SD 6.6] $\mathrm{mL} / \mathrm{kg} / \mathrm{min}$ ). (D) Waist circumference was reduced by a mean 1.3 (SD 1.6) in. MRI-PDFF: magnetic resonance imaging proton density fat fraction; $\mathrm{VO} 2$ peak: peak oxygen consumption.

(A)

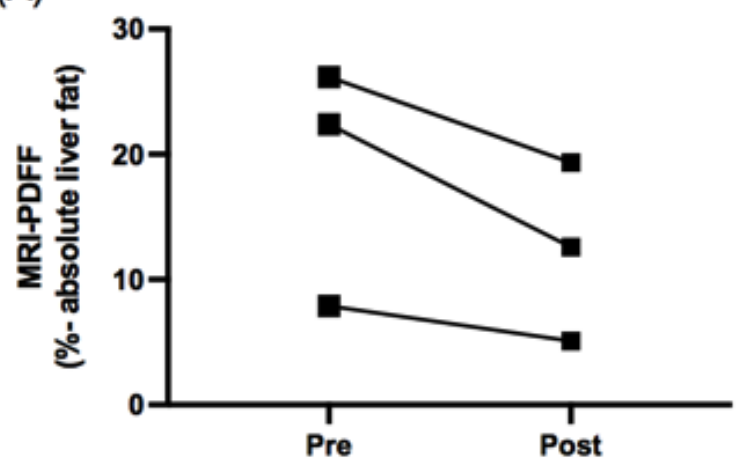

(C)

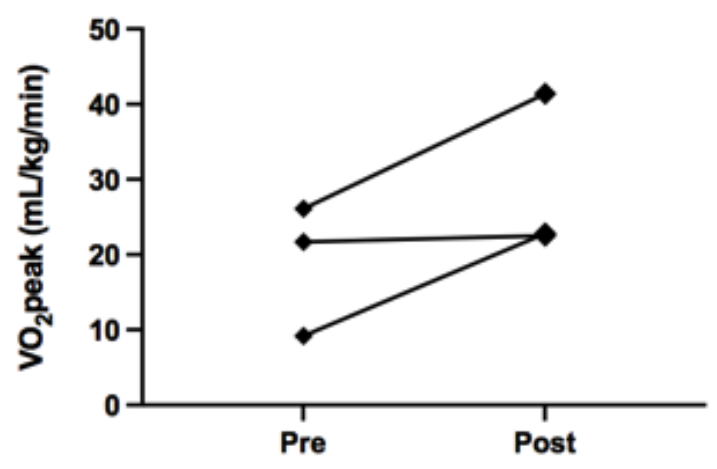

\section{Discussion}

This proof-of-concept study found supervised exercise training delivered via an A-V telehealth platform to be feasible and safe in patients with NASH. All patients met the a priori definition of feasibility and none experienced an adverse event. Importantly, remote exercise training also appears to be efficacious in patients with NASH. The observed reduction in MRI-PDFF-measured liver fat, insulin resistance, body weight, and body fat in parallel with gains in physical fitness even exceed those that are published for supervised in-person exercise training programs of similar length [2]. While we look to future large-scale studies to validate the efficacy of this small study, these data are nonetheless promising and further suggest that $\mathrm{A}-\mathrm{V}$ telehealth has a role in the routine care of patients with NASH.

While this is the first study to employ remote supervision using real-time $\mathrm{A}-\mathrm{V}$ telehealth technology to deliver an exercise training program in patients with $\mathrm{NASH}$, other studies have used web-based or mobile health (mHealth) to deliver an unsupervised exercise training program to a more heterogenous population of patients with all types of NAFLD [6,7]. Pfirrmann et al [6] enrolled 44 patients with NAFLD into an 8-week
(B)

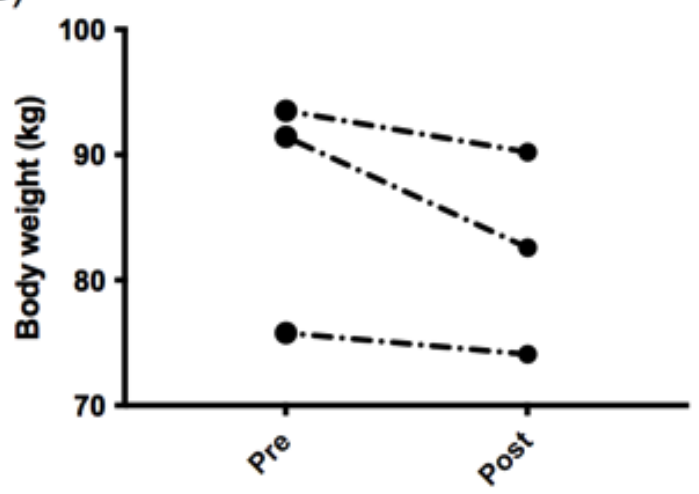

(D)

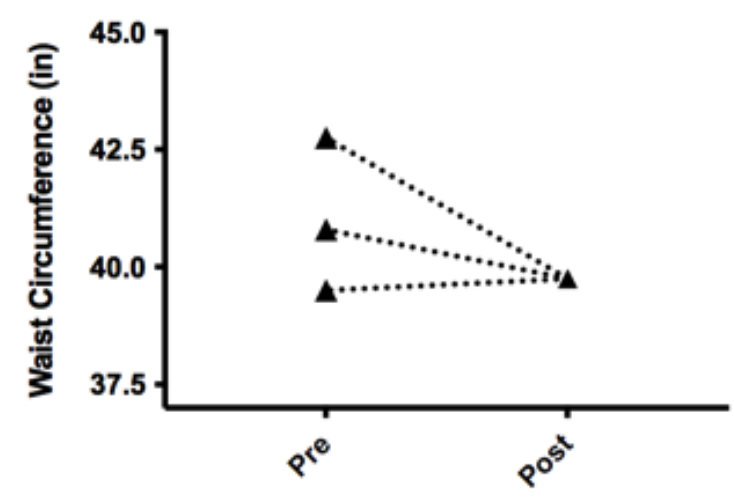

web-based exercise training program, in which patients completed progressive amounts of aerobic and resistance training. While exercise sessions were not directly supervised, weekly feedback was provided to individualize the exercise program and prevent injury. This web-based exercise program was feasible ( $74 \%$ of patients completed $\geq 80 \%$ of the exercise sessions) and safe (no adverse events). Modest gains in physical fitness were seen in parallel with a slight reduction in body weight $(<5 \%)$ and body fat. Changes in liver fat were not reported, although transient elastography was performed, which demonstrated a small reduction in liver stiffness but no change in liver fibrosis stage.

Lim et al [7] recently explored the efficacy of Nutritionist Buddy, an mHealth app-delivered lifestyle intervention, through which patients with NAFLD were given progressive daily step goals up to 10,000 steps/day, on top of real-time dietitian support services for behavioral change. And while the authors did not provide information regarding changes in daily steps or physical activity as a whole, the mHealth intervention was successful in achieving at least 5\% weight loss with corresponding improvement in metabolic parameters in $25 \%$ and $40 \%$ of patients at 3 and 6 months, respectively. However, liver-specific benefits were not measured beyond a reduction in liver enzymes; 
besides, change in liver fat, physical fitness, or insulin resistance were not investigated.

Another important question that remains unanswered is whether or not direct supervision by a fitness professional using telehealth outperforms unsupervised mHealth-based lifestyle modification programs. While it is plausible to imagine regular interaction with a fitness professional over any medium, including $\mathrm{A}-\mathrm{V}$ telehealth technology, may lead to increased accountability and the potential for greater exercise adherence, no direct head-to-head comparison between unsupervised mHealth-based and supervised telehealth exercise training programs has been made to date. Future study investigating this question would seem of high importance given the rapidity at which telehealth is being incorporated into routine medical care and the expected increase in NAFLD and NASH after the COVID-19 pandemic resolves.
Our study has several limitations worth noting: (1) The sample size of 3 patients limits large-scale conclusions. (2) Patients were highly selected and exclusively female, mobile device literate, and English speaking which limits the generalizability of our preliminary findings. (3) While not powered to determine change in clinical outcomes, no secondary clinical outcome achieved statistical significance and future studies are required to confirm our signal of clinical efficacy.

In conclusion, our findings demonstrate proof of concept that exercise training is feasible, safe, and likely efficacious as well. These findings require validation in a larger randomized controlled trial. If validated, new telehealth-delivered exercise training programs have the possibility to increase exercise adherence and sustainability which we would anticipate to alter the natural history of NAFLD and NASH and improve patient-oriented outcomes.

\section{Acknowledgments}

We thank the following individuals for their contributions to the research presented in this manuscript: Chris Sicca and Jeff Vesek at the Penn State Center for NMR Research; Megan Beyer, Breianna Hummer, Zachary Pattison, Gloriany Rivas, and Heather Tressler in the Penn State Division of Gastroenterology and Hepatology; and Kristin Slavoski in the Penn State Physical Medicine \& Rehabilitation Research Laboratory. This grant was funded in part by the NIH grant L30 DK118601. This project is also funded, in part, under a grant with the Pennsylvania Department of Health using Tobacco CURE Funds. The Department specifically disclaims responsibility for any analyses, interpretations, or conclusions. The study was supported by NIH/NCATS grants UL1TR000127 and UL1TR002014.

\section{Authors' Contributions}

VM contributed to manuscript drafting/editing and approval of final version; AF contributed to study conduct, manuscript drafting/editing, and approval of final version; JD contributed to study conduct, manuscript drafting/editing, and approval of final version; BS contributed to study conduct, manuscript drafting/editing, and approval of final version; CS contributed to study conduct, manuscript drafting/editing, and approval of final version; JS contributed to study design, study conduct, data analysis, manuscript drafting/editing, and approval of final version.

\section{Conflicts of Interest}

None declared.

\section{References}

1. Stine JG, Soriano C, Schreibman I, Rivas G, Hummer B, Yoo E, et al. Breaking Down Barriers to Physical Activity in Patients with Nonalcoholic Fatty Liver Disease. Dig Dis Sci 2020 Oct 23:e1. [doi: 10.1007/s10620-020-06673-w] [Medline: 33098023]

2. Thorp A, Stine JG. Exercise as Medicine: The Impact of Exercise Training on Nonalcoholic Fatty Liver Disease. Curr Hepatol Rep 2020 Dec 09;19(4):402-411. [doi: 10.1007/s11901-020-00543-9] [Medline: 33767944]

3. Lin AL, Vittinghoff E, Olgin JE, Pletcher MJ, Marcus GM. Body Weight Changes During Pandemic-Related Shelter-in-Place in a Longitudinal Cohort Study. JAMA Netw Open 2021 Mar 01;4(3):e212536 [FREE Full text] [doi: 10.1001/jamanetworkopen.2021.2536] [Medline: 33749764]

4. Stine JG, Schreibman I, Navabi S, Kang M, Dahmus J, Soriano C, et al. Nonalcoholic steatohepatitis Fitness Intervention in Thrombosis (NASHFit): Study protocol for a randomized controlled trial of a supervised aerobic exercise program to reduce elevated clotting risk in patients with NASH. Contemp Clin Trials Commun 2020 Jun;18:100560 [FREE Full text] [doi: 10.1016/j.conctc.2020.100560] [Medline: 32309672]

5. Kruger C, McNeely ML, Bailey RJ, Yavari M, Abraldes JG, Carbonneau M, et al. Home Exercise Training Improves Exercise Capacity in Cirrhosis Patients: Role of Exercise Adherence. Sci Rep 2018 Jan 08;8(1):99 [FREE Full text] [doi: 10.1038/s41598-017-18320-y] [Medline: 29311671]

6. Pfirrmann D, Huber Y, Schattenberg JM, Simon P. Web-Based Exercise as an Effective Complementary Treatment for Patients With Nonalcoholic Fatty Liver Disease: Intervention Study. J Med Internet Res 2019 Jan 02;21(1):e11250 [FREE Full text] [doi: 10.2196/11250] [Medline: $\underline{\text { 30602434] }}$ 
7. Lim SL, Johal J, Ong KW, Han CY, Chan YH, Lee YM, et al. Lifestyle Intervention Enabled by Mobile Technology on Weight Loss in Patients With Nonalcoholic Fatty Liver Disease: Randomized Controlled Trial. JMIR Mhealth Uhealth 2020 Apr 13;8(4):e14802 [FREE Full text] [doi: 10.2196/14802] [Medline: $\underline{\text { 32281943] }}$

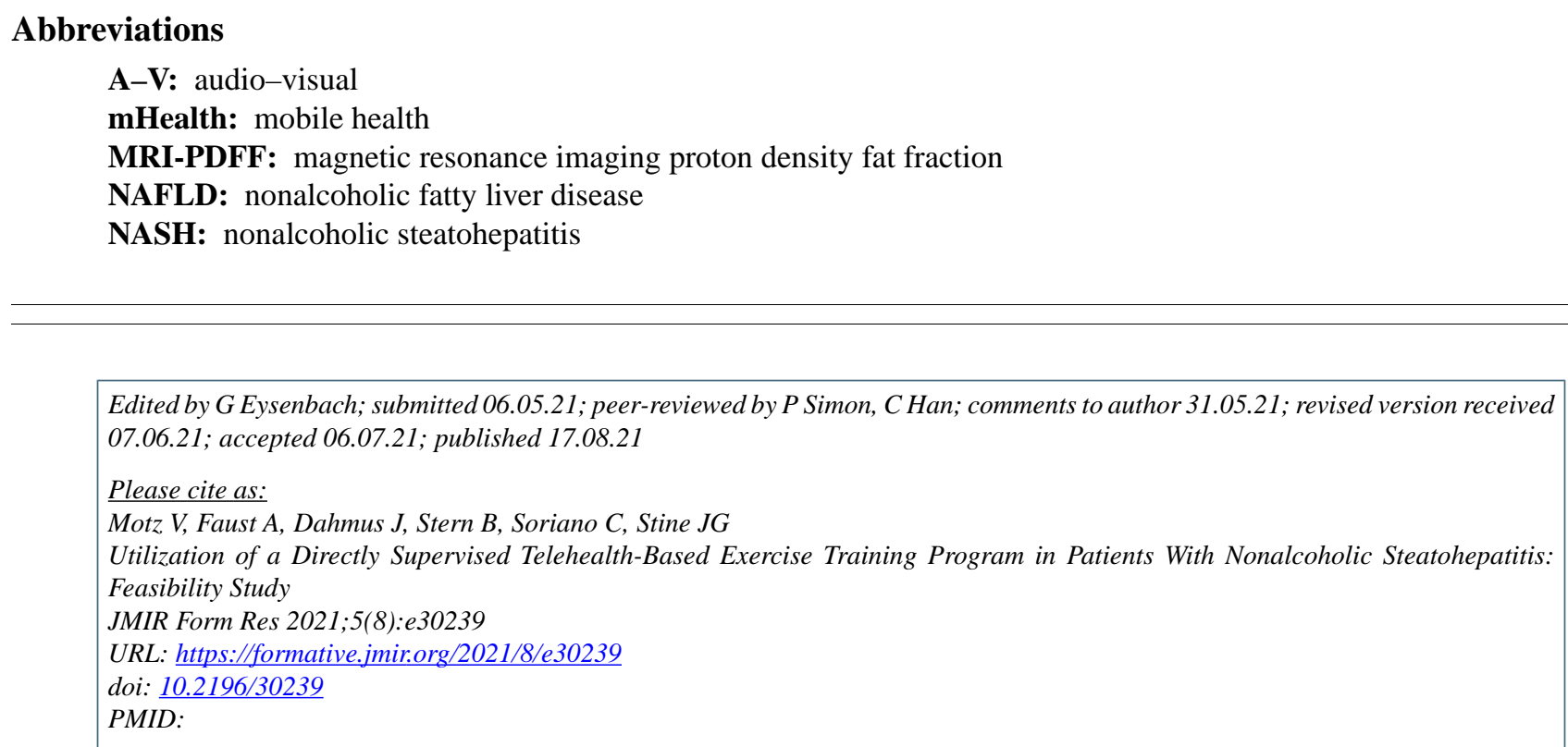

(C) Victoria Motz, Alison Faust, Jessica Dahmus, Benjamin Stern, Christopher Soriano, Jonathan G Stine. Originally published in JMIR Formative Research (https://formative.jmir.org), 17.08.2021. This is an open-access article distributed under the terms of the Creative Commons Attribution License (https://creativecommons.org/licenses/by/4.0/), which permits unrestricted use, distribution, and reproduction in any medium, provided the original work, first published in JMIR Formative Research, is properly cited. The complete bibliographic information, a link to the original publication on https://formative.jmir.org, as well as this copyright and license information must be included. 